\title{
Facoemulsificação por córnea clara no meridiano mais curvo
}

\author{
Phacoemulsification using clear cornea incision in steepest meridian
}

\author{
Fernando Pistarini Gonçalves ${ }^{1}$ \\ Antonio Carlos Lottelli Rodrigues ${ }^{2}$
}

\begin{tabular}{|c|}
\hline RESUMO \\
\hline $\begin{array}{l}\text { Objetivos: Avaliar o efeito da facoemulsificação com incisão em córnea } \\
\text { clara no meridiano mais curvo sobre a magnitude do astigmatismo } \\
\text { ceratométrico pré-operatório. Mapear a magnitude do astigmatismo } \\
\text { cirurgicamente induzido por incisões nas posições nasal }(\mathrm{N}) \text {, temporal } \\
\text { (T), temporal superior(TS) e temporal inferior (TI). Métodos: Foi realizado } \\
\text { estudo ceratométrico prospectivo em } 48 \text { olhos de } 48 \text { pacientes, subme- } \\
\text { tidos a facoemulsificação com incisão do meridiano mais curvo. As } \\
\text { medidas ceratométricas foram realizadas no pré-operatório e um mês } \\
\text { após a realização da cirurgia. O astigmatismo cirurgicamente induzido foi } \\
\text { determinado pelo método das coordenadas retangulares em } 10 \text { passos, } \\
\text { modificado. Resultados: Foram operados } 21 \text { olhos direitos e } 27 \text { olhos } \\
\text { esquerdos e, de acordo com a posição das incisões em córnea clara, foram } \\
\text { divididos em: } 16 \text { olhos } \mathrm{N}, 4 \text { olhos T, } 22 \text { olhos TS, } 6 \text { olhos TI. A média da } \\
\text { ametropia cilíndrica pré-operatória foi de } 1,06 \mathrm{D} \pm 0,65 \text { e a pós-operatória } \\
\text { de } 0,89 \mathrm{D} \pm 0,80 \text {. Houve diminuição estatisticamente significativa no } \\
\text { astigmatismo corneal preexistente (p=0,016). A média total de astigmatismo } \\
\text { cirurgicamente induzido foi de } 0,94 \mathrm{D} \pm 0,56 \text {. Em relação aos grupos o } \\
\text { astigmatismo cirurgicamente induzido foi de } 1,06 \mathrm{D} \pm 0,66 \text { em } \mathrm{N}, 0,87 \mathrm{D} \pm \\
0,20 \text { em T, } 0,95 \mathrm{D} \pm 0,55 \text { em TS e } 0,61 \mathrm{D} \pm 0,25 \text { em TI. Não houve diferença } \\
\text { estatística com relação ao astigmatismo cirurgicamente induzido nos } \\
\text { grupos } \mathrm{N}, \mathrm{T}, \mathrm{TS} \text { e TI ( } \mathrm{p}=0,426 \text { ). Conclusão: A técnica se mostrou efetiva } \\
\text { na redução do astigmatismo ceratométrico pré-operatório. A média do } \\
\text { astigmatismo cirurgicamente induzido foi de } 1,06 \mathrm{D} \pm 0,66 \text { em } \mathrm{N}, 0,95 \mathrm{D} \pm \\
0,55 \text { em TS, } 0,61 \mathrm{D} \pm 0,25 \text { em T e } 0,87 \mathrm{D} \pm 0,20 \text { em TI. }\end{array}$ \\
\hline
\end{tabular}

Descritores: Facoemulsificação; Astigmatismo; Topografia da córnea; Córnea/cirurgia; Ceratocone; Cuidados pré-operatórios; Complicações pós-operatórias

Trabalho realizado no Hospital das Clínicas da Faculdade de Medicina de Botucatu - UNESP.

${ }^{1}$ Residente do terceiro ano de Oftalmologia da Faculdade de Medicina de Botucatu - Universidade Estadual Paulista "Julio Mesquita Filho" - UNESP - Botucatu (SP) - Brasil.

${ }^{2}$ Professor Assistente Doutor, Chefe do Setor de Catarata da Faculdade de Medicina de Botucatu - UNESP Botucatu (SP) - Brasil.

Endereço para correspondência: Depto. OFT/ORL/ CCP. Faculdade de Medicina de Botucatu - UNESP. Distrito de Rubião Júnior, s/n - Botucatu (SP) CEP 18618-970

Recebido para publicação em 16.01.2006

Última versão recebida em 14.08.2006

Aprovação em 06.09.2006

\section{INTRODUÇÃO}

Após a introdução da facoemulsificação por Kelman a indução de astigmatismo corneal, gerados pela extração extra-capsular (EEC), foram quase que totalmente solucionados ${ }^{(1)}$.

A cirurgia de catarata, hoje, é tida como uma cirurgia refrativa, e como tal devemos nos preocupar em corrigir não apenas a ametropia esférica, mas também a cilíndrica ${ }^{(2-3)}$.

Desta forma tem sido proposto o uso de incisões no meridiano mais curvo $^{(3-4)}$ confecção de 2 incisões opostas em córnea clara no meridiano mais curvo $^{(5-6)}$ e ceratotomia astigmática combinada a facoemulsificação ${ }^{(2,7-9)}$ na tentativa de corrigir estas ametropias. As lentes intra-oculares tóricas são uma alternativa para correção do astigmatismo refrativo final ${ }^{(2,7)}$.
} 
A confecção da incisão no meridiano mais curvo (IMC) usa o aplanamento corneal por ela gerado para redução de seu astigmatismo final. Apesar de pequena, a magnitude do astigmatismo provocado pela incisão depende do tamanho e de sua localização ${ }^{(10-1)}$ embora outros não tenham encontrado diferença estatística com incisão em diferentes meridianos ${ }^{(12)}$.

O objetivo do presente trabalho é:

1) Avaliar o efeito da facoemulsificação, através de incisão em córnea clara no meridiano mais curvo (IMC), sobre a magnitude do astigmatismo ceratométrico pré-existente.

2) Mapear a magnitude do astigmatismo cirurgicamente induzido (ACI) pelas incisões nas posições nasal (N), tempo$\operatorname{ral}(\mathrm{T})$, temporal superior (TS) e temporal inferior (TI).

\section{MÉTODOS}

Foi realizado um estudo prospectivo entre fevereiro e junho de 2004, em 48 olhos de 48 pacientes do serviço de oftalmologia da Faculdade de Medicina de Botucatu - UNESP, sendo 22 do sexo feminino e 26 do sexo masculino, com média de idade de 60,02 $\pm 19,30$ anos. Todos apresentavam catarata e astigmatismo ceratométrico maior que $0,50 \mathrm{D}$. Os pacientes com astigmatismo ceratométrico menor ou igual a $0,50 \mathrm{D}$, foram incluídos em outro estudo.

Após a medida ceratométrica automática (Zeiss Acuitus 5051 - Humphrey Instruments), a ecobiometria de contato foi realizada utilizando-se a fórmula SRK/T (Paradigm A/B-Scan Model P37).

Todos os pacientes foram submetidos à cirurgia de facoemulsificação (Alcon - Universal II $^{\circledR}$ ) com anestesia tópica (xilocaína 2\% gel). Uma incisão de 3,0 mm, tunelizada, biplanar, com 1,5 a 1,75 mm de extensão, foi realizada em córnea clara, logo após a arcada vascular e no meridiano mais curvo, utilizando-se lâmina "clear cut" 3,0 Alcon ${ }^{\circledR}$. A incisão auxiliar, com 0,5 a $1,00 \mathrm{~mm}$ de abertura e $1 \mathrm{~mm}$ de extensão, foi confeccionada, também em córnea clara, logo após a arcada vascular e posicionada entre $160^{\circ}$ a $180^{\circ}$ da incisão principal, utilizandose lâmina $15^{\circ} \mathrm{Alcon}^{\circledR}$. Procedeu-se então capsulorrexe com pinça, hidrodisecção e hidrodelineação do núcleo e facoemulsificação utilizando técnicas de "phaco chop, stop-and-chop e pre-slice". Os restos corticais foram aspirados com caneta de irrigação/aspiração automatizada do facoemulsificador. Uma lente intra-ocular de três peças, com parte óptica de acrílico hidrofóbico dobrável de $5,5 \mathrm{~mm}$ de diâmetro e alças de prolene (Type 7B, Alcon ${ }^{\circledR}$ ), foi implantada, no saco capsular, utilizando-se injetor Monarch II Alcon ${ }^{\circledR}$, através da incisão de 3,0 mm. As incisões foram seladas por hidratação com solução salina balanceada. Todas as cirurgias foram realizadas pelos autores.

No dia anterior à cirurgia utilizou-se colírio antiinflamatório não hormonal a cada $8 \mathrm{~h}$ (Still-Allergan ${ }^{\circledR}$ ) que foi mantido até 30 dias de pós-operatório. Foi também prescrito no pós-operatório a associação de dexametasona e tobramicina (TobradexAlcon ${ }^{\circledR}$ ) de $3 / 3 \mathrm{~h}$ no primeiro dia de pós-operatório, $4 / 4 \mathrm{~h}$ do $2^{\circ}$ ao $7^{\circ}$ pós-operatório e $6 / 6 \mathrm{~h}$ até o $30^{\circ}$ pós-operatório.

Nova ceratometria foi realizada, utilizando-se o mesmo ceratômetro, 30 dias após o procedimento.
As médias da ametropia cilíndrica corneal e desvios-padrão pré- e pós-operatórias foram estatisticamente analisados entre si.

A amostra foi dividida em grupos segundo a localização da incisão: T, N, TS e TI. Como ambos cirurgiões são destros, os olhos direitos com meridiano mais curvo entre $90^{\circ} \mathrm{e} 180^{\circ}, 180^{\circ} \mathrm{e}$ entre $0^{\circ}$ e $90^{\circ}$, receberam incisões respectivamente TS, T e TI, os olhos esquerdos com meridianos mais curvos entre $90^{\circ}$ e $180^{\circ}$, $0^{\circ}$ e $90^{\circ}$, receberam incisões respectivamente $\mathrm{Ne} \mathrm{TS}$, procurando posicioná-las exatamente no meridiano mais curvo. O ACI nos diferentes grupos foi calculado através do método das coordenadas retangulares, modificado em 10 passos $^{(13)}$. Os passos foram colocados em uma planilha do Microsoft Excel, e suas médias e desvios-padrão estatisticamente analisados.

No tratamento estatístico do astigmatismo cirurgicamente induzido, foi realizada análise de variância para delineamento inteiramente casualizado com os quatro grupos e número diferente de repetições. Para verificar a diferença entre eles foi aplicado o teste $\mathrm{F}$ ao $\mathrm{n}$. Para verificar diferenças entre os momentos pré- e pós-operatório com relação a ametropia cilíndrica e a ceratometria, foi utilizado teste $T$ pareado para amostras dependentes ao nível de significância $\alpha=0,05$ e o teste de Wilcoxon pareado para amostras dependentes ao nível de significância de $\alpha=0,05$.

\section{RESULTADOS}

Foram operados 21 olhos direitos e 27 olhos esquerdos e de acordo com a posição das incisões em córnea clara foram divididos da seguinte forma: 16 olhos N, 4 olhos T, 22 olhos TS, 6 olhos TI.

A média da ametropia cilíndrica pré-operatória foi de 1,06 \pm $0,65 \mathrm{D}$ e pós-operatória de $0,89 \pm 0,80 \mathrm{D}$. Houve diminuição estatisticamente significativa na ametropía cilíndrica pós-operatória $(\mathrm{p}=0,016)($ Gráficos 1 e 2).

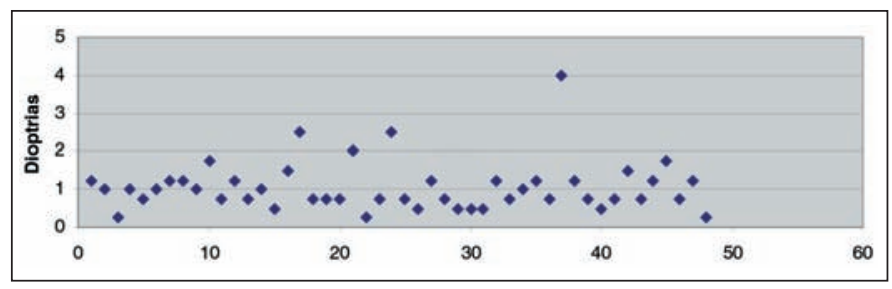

Gráfico 1 - Distribuição dos astigmatismos corneais pré-operatórios

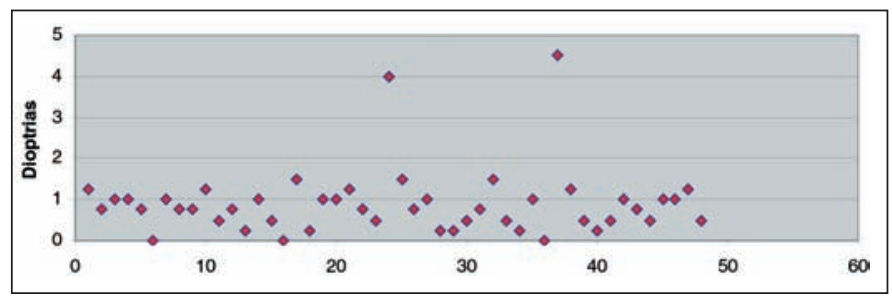

Gráfico 2 - Distribuição dos astigmatismos corneais pós-operatórios 
A média total de ACI foi de $0,94 \mathrm{D} \pm 0,56$. Em relação aos grupos o ACI foi de 1,06 $\pm 0,66 \mathrm{D} \mathrm{em} \mathrm{N}, 0,87 \pm 0,20 \mathrm{D} \mathrm{em} \mathrm{T}, 0,95$ $\pm 0,55 \mathrm{D}$ em TS e $0,61 \pm 0,25 \mathrm{D}$ em TI. Não encontramos diferença estatística entre os grupos $(\mathrm{p}=0,426)$. Em média, todos os grupos mostraram aplanamento em meridiano do quadrante incisional de $-0,46 \pm 0,40 \mathrm{D}$ e encurvamento no meridiano oposto de $+0,47 \pm 0,34 \mathrm{D}$.

A ceratometria média pré-operatória foi de $44,04 \mathrm{D} \pm 1,30 \mathrm{e}$ pós-operatória de 44,01 $\mathrm{D} \pm 1,29$, não há diferença estatística entre elas $(\mathrm{p}=0,569)$.

\section{DISCUSSÃO}

Existem inúmeros trabalhos na literatura reportando os efeitos da incisão para facoemulsificação em córnea clara e o aplanamento corneano gerado no meridiano da incisão ${ }^{(10,12)}$.

Como pudemos observar não ocorre apenas um aplanamento no meridiano da incisão, mas também um encurvamento do meridiano oposto, ambos praticamente de mesma magnitude.

Aproveitando este efeito, alguns autores utilizando a IMC de $4 \mathrm{~mm}$ descreveram decréscimo na média do astigmatismo ceratométrico pré-operatório de $0,96 \mathrm{D} \pm 0,03$ para $0,78 \mathrm{D} \pm 0,06$ com 6 meses de pós-operatório ${ }^{(14)}$.

Alguns autores ${ }^{(15)}$ encontraram redução estatisticamente significativa de $0,48 \mathrm{D} \pm 0,6 \mathrm{em}$ pacientes IMC de $3,5 \mathrm{~mm}$, assim como descrito por outros autores ${ }^{(16)}$. Alguns autores defendem o posicionamento da incisão no meridiano mais curvo a fim de prevenir o astigmatismo pós-operatório em córneas com ametropia cilíndrica entre 0,50 e 1,2 D, no entanto conclui que o método é insuficiente para correção de córneas com astigmatismo maior ${ }^{(17)}$.

Em nosso estudo houve decréscimo de 0,17 D na média do astigmatismo ceratométrico pré-operatório, tal redução foi estatisticamente significativa e bastante semelhante à encontrada por alguns autores ${ }^{(14)}$.

Em relação ao ACI, ele foi em média de $0,94 \mathrm{D} \pm 0,56$ e apesar da incisão nasal ter induzido astigmatismo de maior magnitude, não encontramos diferença estatística entre os grupos T, TS, TI e N encontraram médias de ACI de 1,17 D após 6 semanas e de 1,04 D após 1 ano com incisões horizontais de $3,5 \mathrm{~mm}$, sendo temporais em olho direito e nasais em olho esquerdo. A média de $\mathrm{ACI}^{(10)}$ com 6 semanas de pósoperatório foi de $0,74 \mathrm{D}$ em incisões temporais e de 1,65 em incisões nasais, esta tendência persistiu após 12 meses de cirurgia com médias de 0,71 nas incisões temporais e 1,41 nas nasais. As diferenças em relação à posição da incisão foram estatisticamente significativas.

Usando incisões de 3,2 mm não encontraram diferenças estatísticas no ACI em incisões temporal superior e nasal superior com até 1 ano de pós-operatório ${ }^{(12)}$.

Alguns autores não encontraram diferenças estatisticamente significativas no ACI por incisões temporais de $3,2 \mathrm{~mm}$ em córnea clara, com 5, 30 e 90 dias de pós-operatório ${ }^{(18)}$.
As incisões nasais tendem a gerar maior astigmatismo, pois se encontram mais próximas do eixo visual que as temporais $^{(11)}$, apesar de nosso grupo nasal ter gerado um ACI maior, assim como outros ${ }^{(12)}$ não conseguimos diferença estatística entre as diferentes posições da incisão, muito provavelmente pelo número pequeno da amostra nos grupos.

Seria de se esperar que a média de redução ceratométrica $(0,17 \mathrm{D})$ fosse igual à média do $\mathrm{ACI}(0,94 \mathrm{D})$ já que, teoricamente, a incisão foi posicionada a fim de induzi-lo exatamente nos eixos mais curvos. Se por exemplo tivéssemos um astigmatismo de $-1,00 \mathrm{D}$ contra a regra $\left(90^{\circ}\right)$ e ao posicionarmos uma incisão a $180^{\circ}$ graus fossemos capaz de induzir um astigmatismo (ACI) de $-1,00 \mathrm{D}$ a favor da regra, exatamente a $180^{\circ}$, o astigmatismo final seria zero. No entanto se no mesmo exemplo, a incisão fosse colocada $10^{\circ}$ distante do eixo $180^{\circ}$ (a $170^{\circ}$ ) restaria um astigmatismo de $-0,35 \mathrm{D}$, porém no eixo $130^{\circ}$, se distanciarmos agora $20^{\circ}$ do eixo $180^{\circ}\left(160^{\circ}\right)$ o astigmatismo residual seria de $-0,68 \mathrm{D}$ a $125^{\circ}$ e assim por diante. Da mesma forma supondo uma córnea com astigmatismo de -1,06 D (média do astigmatismo pré-operatório encontrado) se fossemos capaz de com a incisão gerar um ACI de -0,94 D (média do ACI encontrado) exatamente no meridiano que desejássemos, teríamos um astigmatismo pós-operatório residual de $-0,12 \mathrm{D}$ (1,06 D - 0,94 D). No entanto nossa média de astigmatismo pós-operatório final foi de -0,89 D. Continuando com o exemplo acima, se posicionarmos agora, a incisão $26^{\circ}$ distante do meridiano desejado, teremos um astigmatismo residual de 0,88 , isto é, muito próximo da média do astigmatismo pósoperatório que obtivemos. Poderíamos dizer então, baseados nas análises vetoriais, que a incisão gerou um ACI em média $26^{\circ}$ a $27^{\circ}$ distante do meridiano que desejávamos.

Na realização das incisões não fizemos marcações corneais e isto é um fator de erro, temos que considerar ainda que a incisão de $3,00 \mathrm{~mm}$ ocupa aproximadamente $30^{\circ}$ de arco. Outro fator que pode ainda contribuir para o erro é o estresse cirúrgico que pode sofrer uma das extremidades da incisão, principalmente nas incisões nasais quando na tentativa de evitar o nariz, o cirurgião tende a tracionar o olho para cima ${ }^{(11)}$. Acreditamos que se posicionássemos melhor a incisão, usando marcadores, e evitássemos o estresse cirúrgico na incisão conseguiríamos resultados significativos na redução astigmática, pois estaríamos assim, aproveitando o benefício máximo do ACI.

A mudança de posicionamento do cirurgião de acordo com a localização da incisão se constitui em uma desvantagem na técnica.

\section{CONCLUSÃO}

1) A facoemulsificação, através de incisão em córnea clara no meridiano mais curvo reduz o astigmatismo ceratométrico pré-existente.

2) Para a amostra estudada o ACI foi de $1,06 \mathrm{D} \pm 0,66 \mathrm{em} \mathrm{N}$, $0,87 \mathrm{D} \pm 0,20 \mathrm{em} \mathrm{T}, 0,95 \mathrm{D} \pm 0,55 \mathrm{em}$ TS e $0,61 \mathrm{D} \pm 0,25 \mathrm{em} \mathrm{TI}$. Não houve diferença estatística entre eles. 


\section{ABSTRACT}

Purpose: To evaluate the effect of phacoemulsification through clear cornea incision in the steepest meridian (ISM), on the magnitude of preoperative keratometric astigmatism. To map the magnitude of surgically induced astigmatism (SIA) by clear cornea incision in the positions: nasal (N), temporal (T), superior temporal (ST) and inferior temporal (IT). Methods: A prospective keratometric study was performed in 48 eyes of 48 patients, submitted to phacoemulsification by incision in the steepest meridian. Keratometric measures were taken preoperatively and one month after surgery. Surgically induced astigmatism was determined by the rectangular coordinate by the modified ten-step method. Results: There were 21 right eyes and 27 left eyes submitted to surgery, 16 eyes being N, 4 eyes T, 22 eyes TS, and 6 eyes TI. The average preoperative and postoperative cylindrical ametropias were, respectively, $1.06 \pm 0.65 \mathrm{D}$ and $0.89 \pm 0.80 \mathrm{D}$. We found a statistical difference regarding decrease in the preoperative keratometric astigmatism $(\mathrm{p}=0.016)$. The total average of the surgically induced astigmatism was $0.94 \pm 0.56 \mathrm{D}$. In relation to the groups, surgically induced astigmatism was $1.06 \pm 0.66 \mathrm{D}$ in N, $0.87 \pm$ $0.20 \mathrm{D}$ in T, $0.95 \pm 0.55 \mathrm{D}$ in TS and $0.61 \pm 0.25 \mathrm{D}$ in TI. There was no statistical difference in the surgically induced astigmatism between these groups $(\mathrm{p}=0.426$ ). Conclusion: The technique was shown to be effective in decreasing the preoperative keratometric astigmatism. The surgically induced astigmatism was $1.06 \pm 0.66 \mathrm{D}$ in N, $0.95 \pm 0.55 \mathrm{D}$ in TS, $0.61 \pm 0.25 \mathrm{D}$ in T and $0.87 \pm 0.20 \mathrm{D}$ in TI.

Keywords: Phacoemulsification; Astigmatism; Corneal topography; Cornea/surgery; Keratoconus; Preoperative care; Postoperative complications

\section{REFERÊNCIAS}

1. Kelman CD. Phaco-emulsifiction and aspiration. A new technique of cataract removal. A preliminary report. Am J Ophthalmol. 1967;64(1):23-35.
2. Kohnenen T, Koch DD. Methods to control astigmatism in cataract surgery. Curr Opin Ophthalmol. 1996;7(1):75-80.

3. Nielsen PJ. Prospective evaluation of surgically induced astigmatism and astigmatic keratotomy effects of varous self-seling small incisions. J Cataract Refract Surg. 1995;21(1):43-8.

4. Pfleger T, Skorpik C, Menapeace R, Scholz U, Weghaupt H, Zehetmayer M. Long-term course of induced astigmatism after clear corneal incision cataract surgery. J Cataract Refract Surg. 1996;22(1):72-7.

5. Lever J, Dahan E. Opposite clear corneal incisions to correct pre-existing astigmatism in cataract surgery. J Cataract Refract Surg. 2000;26(6):803-5. Comment in: J Cataract Refract Surg. 2000;26(12):1697-8. J Cataract Refract Surg. 2000;26(6):789-90. J Cataract Refract Surg. 2001;27(1):7-8.

6. Tadros A, Habib M, Tejwani D, Von Lany H, Thomas P. Opposite clear corneal incisions on the steep meridian in phacoemulsification: early effects on the cornea. J Cataract Refract Surg. 2004;30(2):414-7.

7. Gills JP. Treating the astigmatism at the time of cataract surgery. Curr Opin Ophthalmol. 2002;13(1):2-6.

8. Kershner RM. Keratolenticuloplasty: arcuate keratotomy for cataract surgery and astigmatism. J Cataract Refract Surg. 1995;21(3):274-7. Comment in: J Cataract Refract Surg. 1995;21(6):597-8.

9. Akura J, Matsuura K, Hatta S, Kaneda S, Kadonosono K. Clinical application of full-arc, depth-dependent, astigmatism keratotomy. Cornea. 2001;20(8):839-43.

10. Barequet IS, Yu E, Vitale S, Cassard S, Azar DT, Stark WJ. Astigmatism outcomes of horizontal temporal versus nasal clear corneal incision cataract surgery. J Cataract Refract Surg. 2004;30(2):418-23.

11. Kohnen S, Neuber R, Kohnen T. Effect of temporal and nasal unsutured limbal tunnel incisions on induced astigmatism after phacoemulsification. J Cataract Refract Surg. 2002;28(5):821-5.

12. Ermis SS, Inan UU, Ozturk F. Surgically induced astigmatism after superotemporal and superonasal clear corneal incisions in phacoemulsification. J Cataract Refract Surg. 2004;30(6):1316-9.

13. Holladay JT, Cravy TV, Koch DD. Calculating the surgically induced refractive change following ocular surgery. J Cataract Refract Surg. 1992;18(5):429-43. Comment in: J Cataract Refract Surg. 1993;19(2):319-20. J Cataract Refract Surg. 1993;19(2):320-2.

14. Zemaitiene R, Jasinskas V, Januleviciene I. [Correction of corneal astigmatism during phacoemulsification]. Medicina (Kaunas). 2003;39(12):1175-83. Lithuanian. Erratum in: Medicina (Kaunas). 2004;40(1):93.

15. Titiyal JS, Baidya KP, Sinha R, Ray M, Sharma N, Vajpayee RB, et al. Intraoperative arcuate transverse keratotomy with phacoemulsification. J Refract Surg. 2002;18(6):725-30.

16. Xie L, Zhu G, Wang X. [Clinical observation of astigmatism induced by corneal incision after phacoemulsification]. Zhonghua Yan Ke Za Zhi. 2001; 37(2):108-10. Chinese.

17. Matsumoto Y, Hara T, Chiba K, Chikuda M. Optimal incision sites to obtain an astigmatism-free cornea after cataract surgery with a $3.2 \mathrm{~mm}$ sutureless incision. J Cataract Refract Surg. 2001;27(10):1615-9.

18. Crema AE, Walsh A, Yamame R. Astigmatismo induzido após facoemulsificação através de incisões auto-selantes escleral superior e corneana temporal. Arq Bras Oftalmol. 1998;61(1):96-9.

\section{Ao enviar um artigo para publicação, leia ATENTAMENTE as instruções para autores, constante no final de cada fascículo.}

